\title{
Development of a Fast, Sensitive and Robust LC-MS/MS Method for the Analysis of Natamycin in Wine
}

\author{
P. Alberts ${ }^{1,2}$, M.A. Stander ${ }^{3}$, A. de Villiers ${ }^{1 *}$
}

(1) Department of Chemistry and Polymer Science, Stellenbosch University, Private Bag X1, Matieland 7602 (Stellenbosch), South Africa

(2) National Department of Agriculture, Forestry and Fisheries, Private Bag X5015, Stellenbosch 7600, South Africa

(3) Central Analytical Facility, Stellenbosch University, Private Bag X1, Matieland 7602 (Stellenbosch), South Africa

Submitted for publication: July 2010

Accepted for publication: September 2010

Key words: Natamycin, wine, ultra-high pressure liquid chromatography (UHPLC), electrospray tandem quadrupole mass spectrometry (ESI-MS/MS), solid phase extraction (SPE)

\begin{abstract}
Natamycin is a naturally occurring antifungal agent used to inhibit the growth of moulds and yeasts. Characteristics such as low effective concentrations, broad applicability and neutral flavour impact make this compound an ideal preservative. Although widely used in the global food industry, natamycin is forbidden in wine in the European Union (EU). It is a permitted wine additive in South Africa, although export wines must comply with EU standards. Germany in particular implemented strict measures to prevent wines containing natamycin from entering their market. Sensitive analytical methodologies with the capacity to regulate this segment of the wine export industry are therefore required. Here we report on the development of a simple, robust and fast liquid chromatography-electrospray ionisation tandem mass spectrometric (LC-ESI-MS/MS) method for the determination of natamycin in wine. Sample cleanup involves dilution followed by direct elution of natamycin from aminopropyl SPE cartridges. Recoveries are better than $\mathbf{8 0} \%$ relative standard deviation (RSD $<10 \%$ ), while the limit of detection (LOD) of the method is $0.0003 \mathrm{mg} / \mathrm{L}$, which renders it compliant with EU standards. The method also yields qualitative information for positive compound identification. The degradation of natamycin in wine was also studied and kinetic parameters are reported in this matrix for the first time. The activation energy for the decomposition reaction is $\sim 80 \mathrm{~kJ} / \mathrm{mol}$ and the half-life under normal wine storage conditions is in the order of 20 days. As in the wine matrix, natamycin in aqueous calibration standards are labile, necessitating regular preparation and cold storage of standard solutions to ensure accurate quantitation.
\end{abstract}

\section{INTRODUCTION}

Natamycin, also known as pimaricin, is a macrolide polyene antifungal agent that specifically inhibits the growth of moulds and yeasts (Fig. 1) (Budavari, 1989; Food Standards Australia New Zealand, 2004). Natamycin occurs naturally and is produced during fermentation by the bacterium Streptomyces natalensis, commonly found in soil. Due to the amphiphilic nature of the molecule it has low solubility in water, but it is effective at low concentrations, exhibits a wide spectrum of activity and has a neutral flavour impact, characteristics which render it ideally suited as a preservative (Budavari, 1989; Food Standards Australia New Zealand, 2004). While natamycin and its metabolites lack acute toxicity, its use as a preservative should be considered in the light of its potential for antimicrobial resistance (World Health Organization, 2002; Food Standards Australia New Zealand, 2004).

Natamycin is permitted as an antimicrobial preservative in more than 70 countries, mainly for processed meat and cheese products. In South Africa it is allowed as a preservative in a wide range of products, including wine (Food Standards Australia New Zealand, 2004). However, South African legislation explicitly forbids its use in liquor products intended for export to markets where it is prohibited (Republic of South Africa, 1989). Since natamycin is forbidden in wine in the European Union (EU), wine containing this compound may not be exported to that market. In order to ensure accessibility to the important EU market ( $\sim \mathrm{R} 18$ billion annually; Department of Agriculture, Forestry and Fisheries) for South African exporters, it is imperative that methodologies be developed to demonstrate the compliance of export liquor products with relevant EU directives.

Natamycin is routinely analysed in a wide variety of foodstuffs, where its use is allowed, by spectrophotometric methods or a simple extraction step followed by reversed phase high performance liquid chromatography (HPLC) (De Ruig et al., 1987; Capitan-Vallvey et al., 2000). However, neither of these methods provides the required sensitivity or selectivity for the analysis of this compound

*Corresponding author: ajdevill@sun.ac.za

Aknowledgements: The authors gratefully acknowledge financial support from Winetech 
in the complex wine matrix at the regulatory levels. EU regulations stipulate that natamycin should be absent from wine, therefore regulatory levels are dictated by the capabilities of the analytical methods. Currently a level of $<0.005 \mathrm{mg} / \mathrm{L}$ is enforced in Germany (personal communication from German authorities). Therefore, the objective of this study was, in the first instance, the development of rapid, sensitive and robust methodologies for the determination of natamycin in wine. In view of the high-throughput requirement, methodologies based on rapid sample preparation and fast analysis using ultrahigh pressure liquid chromatography (UHPLC) technology were developed. Specifically, the known benefits of small particlepacked columns and elevated pressure operation for fast LC separations were combined with the high selectivity inherent in tandem mass spectrometric analysis to ensure fast and accurate determination of the target analyte (De Villiers et al., 2006). As part of this research, the degradation kinetics of natamycin in different matrices were investigated in view of the relevance of the known instability of this compound (Brik, 1976; Koontz et $a l ., 2003)$ to its accurate analytical determination.

\section{MATERIALS AND METHODS \\ Chemicals and standards}

High purity chemicals and solvents were used throughout, except for natamycin, which was a commercial grade mixture of 50\% natamycin in glucose. Methanol was from Merck (Darmstadt, Germany), acetonitrile, ammonium formate, ethanol, erioglaucine, sodium hydroxide and tartaric acid were from Sigma-Aldrich (Mulbarton, South Africa), and formic acid was from Saarchem (Wadeville, South Africa). C18 solid phase extraction (SPE) cartridges $(500 \mathrm{mg} / 3 \mathrm{~mL})$ were from Agilent Technologies (Chemetrix, South Africa) and aminopropyl SPE cartridges $(500 \mathrm{mg} / 3 \mathrm{~mL})$ were from Waters Corporation (Microsep, South Africa).

A natamycin stock standard was standardised spectrophotometrically using published molar absorptivity data (Budavari, 1989) and the purity of the reference material was calculated from this information. Working standards were prepared by serial dilution of the stock standard with a solution of $40 \%$ methanol in water containing $0.5 \%$ formic acid. Wine samples for the recovery and degradation studies were fortified volumetrically by adding appropriate amounts of reference standards. The recovery of natamycin was determined by quantitative analysis of samples fortified at three levels with known amounts prior to sample preparation $(n=18)$. In the degradation study, the amount of natamycin remaining was quantified relative to an erioglaucine standard, which remained completely stable for the duration of the study.

\section{Sample preparation}

The optimised C18 SPE sample cleanup procedure consisted of pre-conditioning the cartridge consecutively with $3 \mathrm{~mL}$ each of methanol and water. An aliquot of $2 \mathrm{~mL}$ of wine was loaded, followed by matrix removal with $3 \times 1 \mathrm{~mL}$ of $40 \%$ methanol and $0.5 \%$ formic acid in water. Natamycin was eluted $(3 \times 0.7$ $\mathrm{mL}$ ) with a solution of $60 \%$ acetonitrile and $0.5 \%$ formic acid in water. The effluent was collected in a $5 \mathrm{~mL}$ graduated tube. Typical volumes recovered were approximately $1.9 \mathrm{~mL}$, which were then reconstituted to $2 \mathrm{~mL}$ with water.

The aminopropyl-based SPE procedure consisted of mixing together, in a small test tube, $0.8 \mathrm{~mL}$ wine and $0.8 \mathrm{~mL}$ of a solution of $50 \%$ methanol and $1 \%$ formic acid in water. The diluted sample was then passed through the SPE cartridge without any pre-conditioning. The effluent $(\sim 1 \mathrm{~mL})$ was collected directly into a $1.8 \mathrm{~mL}$ autosampler vial for analysis.

\section{Liquid chromatographic methods and instrumentation}

An Agilent 1100 LC system (Agilent Technologies) fitted with quaternary pump, autosampler, column oven and UVvisible diode array detector was used for the LC-UV analyses. Separations were performed in reversed phase mode employing a Phenomenex Luna phenyl-hexyl column ( 150 x $4.6 \mathrm{~mm}, 3 \mu \mathrm{m}$ particle size) at $25^{\circ} \mathrm{C}$. The mobile phase was a $2 \mathrm{mM}$ ammonium formate in acetonitrile (solvent $\mathrm{A}$ ) $/ 2 \mathrm{mM}$ ammonium formate in water (solvent B) gradient. The gradient started at $10 \%$ solvent A, increasing linearly to $70 \%$ in $7.4 \mathrm{~min}$, followed by reequilibration for $2.6 \mathrm{~min}$ (total run-time $10 \mathrm{~min}$ ). The flow rate was $1.0 \mathrm{~mL} / \mathrm{min}$ and variable injection volumes of up to $100 \mu \mathrm{L}$ were used for purified extracts. Spectrophotometric detection was performed at $305 \mathrm{~nm}$, while spectra between 210 and 400 nm were acquired.

For LC-MS/MS, a Waters Acquity UPLC system incorporating a binary pump, vacuum degasser, autosampler, column oven and Micromass Xevo tandem quadrupole mass spectrometric detector was used. Ionisation was performed using electrospray ionisation in positive mode. Samples were separated on a Waters Acquity BEH phenyl-hexyl column $(100 \times 2.1 \mathrm{~mm}, 1.7 \mu \mathrm{m})$ at $50^{\circ} \mathrm{C}$. Variable injection volumes in the partial loop mode were used, with the maximum injection volume limited to $10 \mu \mathrm{L}$. An acetonitrile $/ 0.01 \%$ acetic acid in water gradient was used at a flow rate of $0.3 \mathrm{~mL} / \mathrm{min}$, starting at $5 \%$ acetonitrile isocratic for 18 seconds, increased linearly to $65 \%$ acetonitrile in three min. A 24-second column cleanup step followed, consisting of $95 \%$ acetonitrile at a flow rate of 0.5 $\mathrm{mL} / \mathrm{min}$. Re-equilibration was for $1.5 \mathrm{~min}$ at $0.3 \mathrm{~mL} / \mathrm{min}$ (total run-time five minutes). Natamycin was eluted at $2.80 \mathrm{~min}$ under the described conditions. The column effluent was therefore directed to the detector between 2.2 and $3.2 \mathrm{~min}$ only, with the rest of the chromatographic run being vented to waste. The following multiple reaction monitoring (MRM) transitions were acquired, with the corresponding optimal collision energies given in parenthesis: $m / z=666.1 \rightarrow 648.0(10 \mathrm{eV}), 503.0(10$ $\mathrm{eV}), 485.0(14 \mathrm{eV})$ and $467.0(12 \mathrm{eV})$. The ion $\mathrm{m} / \mathrm{z}=503.0$<smiles>CC(CC1OC(C)C(O)C(N)C1O)OC(=O)/C=C/C1OC1CC(O)CC1(O)CC(O)C(C(=O)O)C(CC2/C=C/C=C/C=C/C=C/CC(C)C(O)C(C)C3OC3O2)O1</smiles>

FIGURE 1

Chemical structure of natamycin $\left(\mathrm{C}_{33} \mathrm{H}_{47} \mathrm{NO}_{13}\right)$. 
was the most intense transition and was therefore used as the quantifier, while $\mathrm{m} / \mathrm{z}=485.0$ served as confirmatory qualifier. The source parameters were as follows: capillary voltage 3.7 $\mathrm{kV}$, cone voltage $16 \mathrm{~V}$ and extractor voltage $3 \mathrm{~V}$. The source and desolvation temperatures were $120^{\circ} \mathrm{C}$ and $350^{\circ} \mathrm{C}$ respectively, while the desolvation and cone gas flows were 600 and $60 \mathrm{~L} / \mathrm{h}$, respectively.

\section{RESULTS AND DISCUSSION}

\section{Sample preparation}

Solid phase extraction (SPE) is a versatile and efficient sample preparation technique offering a multitude of separations based on polar, hydrophobic and/or ion exchange interactions (Thurman et al., 1998). Two SPE sample preparation strategies were developed and evaluated for suitability in this application. Sample pre-concentration was not pursued; instead, the emphasis was on the removal of potential interferences while maintaining the matrix in a weak solvent relative to the intended separation in order to utilise the large loading capacity inherent to $\mathrm{LC}$ to achieve low detection levels.

Natamycin is hydrophobic and is retained strongly in reversed phase mode on $\mathrm{C} 18 \mathrm{SPE}$ cartridges. In this phase, acetonitrile has a higher eluting strength for natamycin compared to methanol. Selectivity could thus be optimised by affecting matrix removal with a relatively strong methanolbased solution, while the analyte is eluted with a relatively weak acetonitrile-based solution. Recovery of natamycin from a wine matrix with the optimised C18-based procedure was very good $(\sim 90 \%)$. However, wine phenolics are co-extracted under these conditions, as it was observed that the extracts of red wines retained a deep red colour.
In contrast, aminopropyl SPE phases have very little affinity for natamycin in a wine matrix. The ability of this phase to retain wine polyphenols was confirmed by experiments with red wine, in which the retention of phenolics was evident from the retained pigments and their removal from the treated samples. This offers the possibility of using the aminopropyl phase to remove the interfering wine matrix by direct elution of natamycin. The recovery of natamycin from a wine matrix was consistently above $80 \%$, while most visible pigments were removed from a red wine treated in this manner. This approach provided the critical benefit of simpler and faster sample preparation, which is indispensable for the fast, routine analysis of large numbers of samples. In addition, the obtained sample is in a weak solvent compared to the starting conditions of RPLC separations, thus facilitating large injection volumes without sacrificing chromatographic performance.

A comparison of the degree of sample matrix elimination achieved for each of these SPE methods is presented in Fig. 2, in which red wine extracts analysed with LC-UV are compared. UV detection at $305 \mathrm{~nm}$ clearly illustrates that the aminopropyl-based SPE procedure provides a much cleaner extract. This is critical for LC-ESI-MS/MS analysis, since ion suppression effects caused by co-eluting wine matrix elements (following C18 cleanup) are effectively eliminated by the aminopropyl-based SPE procedure. Importantly, the elimination of ion suppression during ionisation facilitated the quantification of natamycin in wines with external standards, obviating the necessity for standard addition or isotope-labelled internal standards for accurate quantitation. This procedure was therefore validated for LC-UV and LC$\mathrm{MS} / \mathrm{MS}$ determination of natamycin in wine.

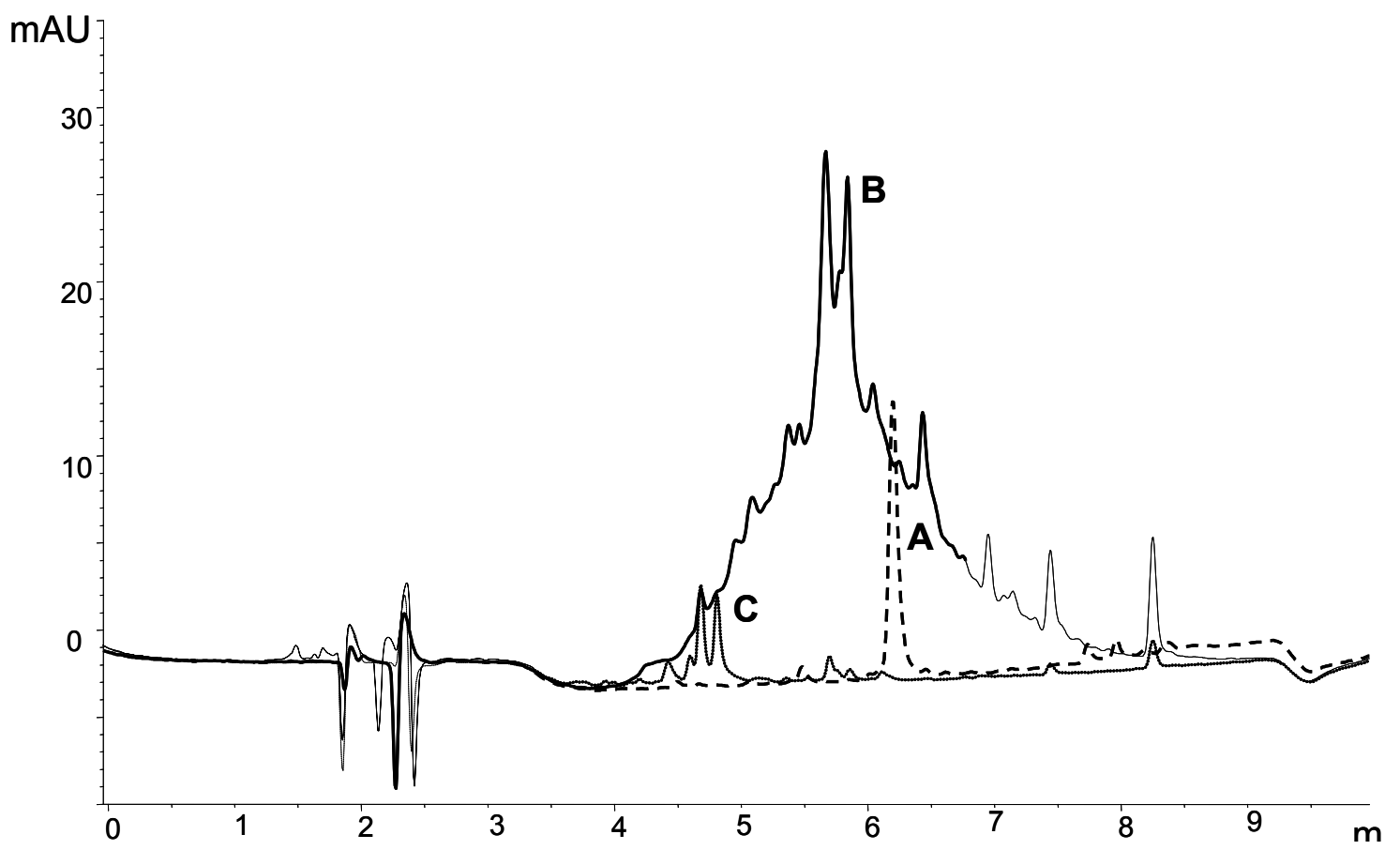

FIGURE 2

Overlay of LC-UV chromatograms of (A) $6.2 \mathrm{mg} / \mathrm{L}$ natamycin standard, (B) red wine sample after cleanup with the C18-based sample preparation procedure and $(\mathrm{C})$ the same wine following aminopropyl SPE cleanup. Injection volumes were $5 \mu \mathrm{L}$ throughout. 


\section{HPLC-UV screening method for natamycin in wine}

Reversed phase separation using a phenyl-hexyl column was employed for the LC-UV and LC-MS/MS determination of natamycin, since better retention was achieved compared to $\mathrm{C} 18$ phases due to the aromatic selectivity offered by the phenyl-hexyl phase. Acetonitrile was selected as the mobile phase organic modifier, since higher separation efficiencies were obtained compared to methanol-based mobile phases. The use of $2 \mathrm{mM}$ ammonium formate ( $\mathrm{pH} 4.28)$ as acidic modifier in the mobile phase improved the chromatographic efficiency.

Natamycin contains strong chromophores and shows five maxima in its UV absorption spectrum (220, 280, 290, 303 and $318 \mathrm{~nm}$ ), with the most intense absorption at $303 \mathrm{~nm}$ displaying a relatively large molar absorptivity of $83220 \mathrm{~L} \cdot \mathrm{mol}^{-1} \cdot \mathrm{cm}^{-1}$ (Budavari, 1989). These spectral features suggest that LC-UV may be applied successfully for the determination of low levels of natamycin. However, wine polyphenols such as flavonols, coumaric acids and anthocyanins also absorb in this region (Abad-Garcia et al., 2009) and are expected to interfere with the LC-UV analysis of natamycin in wine. A sample preparation strategy capable of selectively removing wine polyphenols prior to analysis is therefore indispensable for trace-level analysis.

The optimised aminopropyl SPE sample cleanup procedure was therefore used in combination with LC-UV screening analyses. This procedure was validated. The LC-UV response was linear for injections of 0.1 to $520 \mathrm{ng}$ natamycin on column $(r=0.9999)$. The limit of detection (LOD) and limit of quantitation (LOQ) of the method are $0.1 \mathrm{mg} / \mathrm{L}$ and $0.3 \mathrm{mg} / \mathrm{L}$ respectively for injection volumes of $100 \mu \mathrm{L}$ of purified extracts. Spectral correlation with calibration standards was observed at sample concentrations above $0.3 \mathrm{mg} / \mathrm{L}$. The average recovery of natamycin from wine samples fortified at $10.4 \mathrm{mg} / \mathrm{L}, 1.04$ $\mathrm{mg} / \mathrm{L}$ and $0.208 \mathrm{mg} / \mathrm{L}$ was $89.3 \%(\operatorname{RSD} 9.7 \%, n=18)$.

LC-UV therefore presents a simple, inexpensive and robust methodology for the determination of natamycin in wine in the sub-parts per million range, while the wide availability of these systems makes this an ideal method for the screening of large numbers of samples. This method may be used to quantify natamycin in wines fortified with this compound for preservative purposes (for example wines intended for the local market). However, LC-UV does not meet the criteria to demonstrate conformity to EU regulations for exported wine, and fails to detect lower levels of natamycin such as might result from secondary contamination, for example.

\section{UHPLC-MS/MS method for the trace-level quantitative determination of natamycin in wine}

As the goal of this work was to develop a method suitable for the routine analysis of a large number of samples, an UHPLC column packed with $1.7 \mu \mathrm{m}$ particles was used in order to exploit the well-known benefits of these particles for very fast analyses (De Villiers et al., 2006). A phenyl-hexyl phase was selected for the reasons outlined previously. The use of a 2.1 $\mathrm{mm}$ internal diameter column at an optimal flow rate of $0.3 \mathrm{~mL} /$ min furthermore allows direct connection to ESI-MS without post-column splitting. Positive mode electrospray ionisation (ESI) was used for LC-MS/MS analyses, as atmospheric pressure chemical ionisation (APCI) of this large molecule proved unsuccessful. MS source conditions were optimised by the infusion of a $5 \mathrm{mg} / \mathrm{L}$ standard while adjusting experimental parameters to produce the best sensitivity. It was further established that electrospray ionisation efficiency decreased with increasing levels of acid in the mobile phase. The acid content of the mobile phase was therefore minimised in favour of enhanced sensitivity at the cost of an acceptable reduction in the chromatographic performance. In order to optimise this relationship between the chromatographic efficiency and ionisation efficiency, acetic acid was introduced via the aqueous component of the mobile phase so that a relatively high amount was present initially (which minimised band broadening

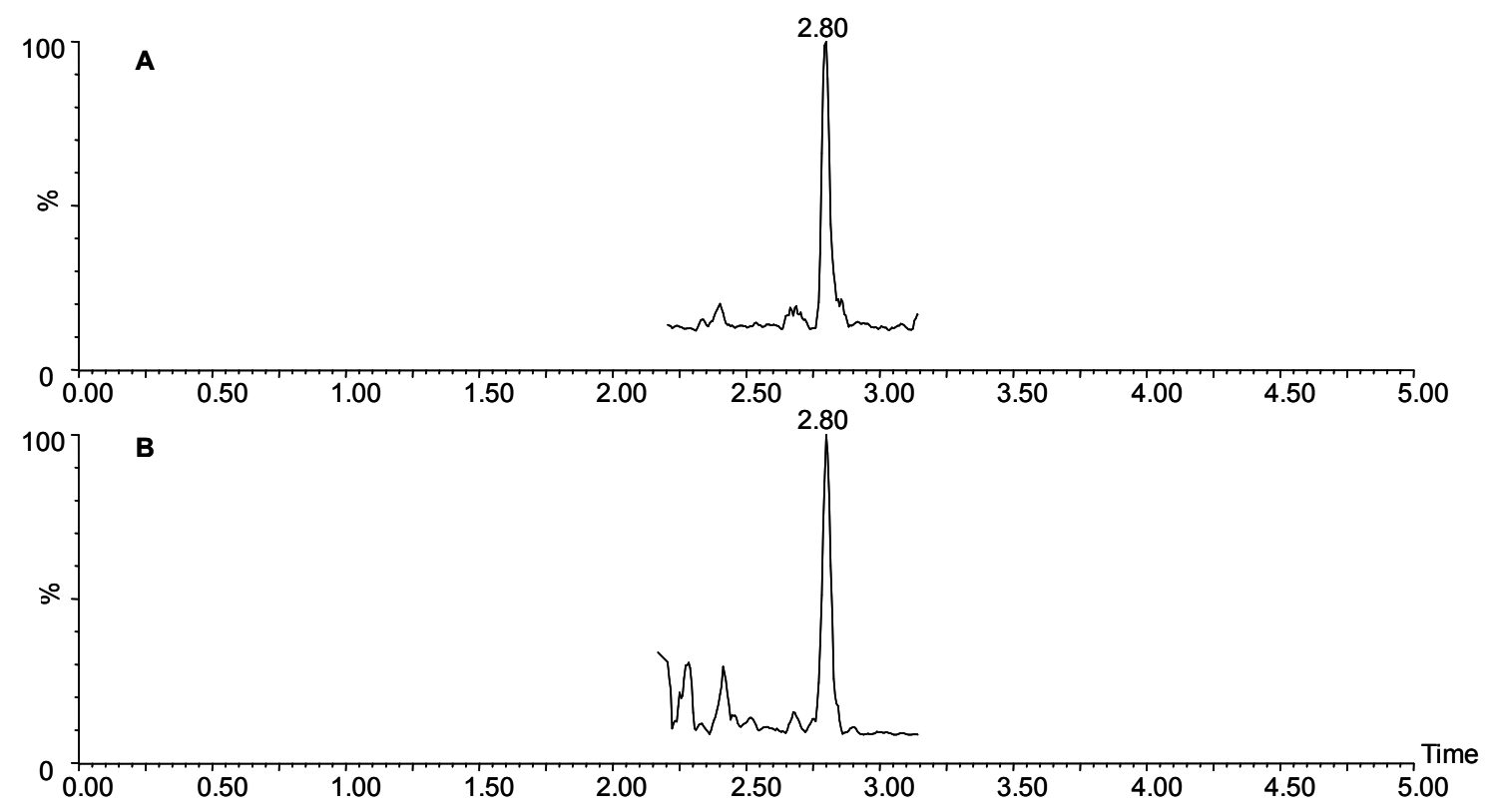

FIGURE 3

Multiple reaction monitoring chromatograms of two ion transitions $(\mathrm{m} / \mathrm{z}=666.1 \rightarrow 485.0(\mathrm{~A})$ and $\mathrm{m} / \mathrm{z}=666.1 \rightarrow 503.0(\mathrm{~B}))$ obtained for the SPE-UHPLC-MS/MS determination of natamycin in a spiked red wine sample containing $0.001 \mathrm{mg} / \mathrm{L}$ (injection volume $10 \mu \mathrm{L}$ ). 
during the initial part of the separation), whereas the analyte was eluted in a fraction of the mobile phase that contained less acid to facilitate ionisation. An example of the optimised SPEUHPLC-ESI-MS/MS analysis of a red wine sample spiked with $0.001 \mathrm{mg} / \mathrm{L}$ natamycin is presented in Fig. 3 .

The overall LC-MS/MS procedure is based on polar SPE sample cleanup, reversed phase chromatographic separation and MS/MS detection in MRM mode, and is therefore characterised by very good selectivity. Moreover, the relatively large molar mass $(\mathrm{m} / \mathrm{z}$ of $\mathrm{M}+1=666.1)$ and high-mass daughter ions $(\mathrm{m} / \mathrm{z}$ 503.0 and 485.0 ) further increase the selectivity of the complete analytical procedure, rendering the method free of interferences (Fig. 3). Validation of the optimised method showed a linear response over the range 0.002 to $20 \mathrm{ng}(\mathrm{r}=0.9999)$. LOD and LOQ values (calculated as the smallest amount of solute that produced a signal equivalent to three and ten times the average noise for unsmoothed chromatograms respectively) were determined as $1.5 \mathrm{pg}$ and $5.0 \mathrm{pg}$ on column, respectively. These values translate to LOD and LOQ values of $0.0003 \mathrm{mg} / \mathrm{L}$ and $0.001 \mathrm{mg} / \mathrm{L}$ respectively, considering that the samples were diluted 1:1 during sample preparation and that injection volumes of $10 \mu \mathrm{L}$ are routinely used. This level of sensitivity is more than required for regulatory purposes (currently enforced at $<$ $0.005 \mathrm{mg} / \mathrm{L}$ by Germany). Identification is based on retention time and two ion transitions $(\mathrm{m} / \mathrm{z}=666.1$ to 503.0 (primary) and 666.1 to 485.0 (secondary)). The ratio of abundance of these transitions (1.6) was used to conclusively identify the analyte.

The accuracy and reproducibility of the method was assessed by determination of the recovery of natamycin from red wine samples fortified to $0.117 \mathrm{mg} / \mathrm{L}, 0.023$ and 0.009 $\mathrm{mg} / \mathrm{L}$. The average recovery of the analytical method was $82.5 \%$ (RSD $6.6 \%, n=18$ ). Method repeatability, evaluated by eight repeated injections of a $0.04 \mathrm{mg} / \mathrm{L}$ standard solution, was $4.1 \%$ RSD for peak area. The intra-assay precision was assessed from the repeatability data obtained in the recovery study. Uncertainty of measurements associated with the LC-MS/
MS procedure was estimated by the $95 \%$ confidence interval about the measurements performed in the recovery study, and was calculated as $\pm 2.6 \%$. As the limit of quantification of the procedure is $0.001 \mathrm{mg} / \mathrm{L}$, and the recoveries of the order 82.5 $\pm 2.6 \%$, the results are reported in units of $\mathrm{mg} / \mathrm{L}$ by recording three significant figures.

\section{Degradation kinetics of natamycin in the wine matrix}

Natamycin is unstable in solution at both low and high $\mathrm{pH}$, and the stability is further influenced by temperature, light exposure and oxidation (Brik, 1976). However, no data on the stability of this compound in wine has been reported. Under acidic conditions (such as encountered in wine), natamycin is degraded rapidly via hydrolysis of the glycosidic bond to yield mycosamine and various other products (Brik, 1976; Pederson, 1992; Koontz et al., 2003). The fact that the compound is labile under the conditions encountered in the wine matrix clearly has implications for the validity of quantitative results, since its concentration is expected to decrease with time. For this reason, the degradation of natamycin was studied in red, white and synthetic wine matrices. The synthetic wine matrix consisted of a solution of $12 \%$ ethanol and $2.5 \mathrm{~g} / \mathrm{L}$ tartaric acid in water $(\mathrm{pH}$ adjusted to 3.5 with sodium hydroxide). Four $\sim 4 \mathrm{~L}$ batches (two red, one white and one synthetic) were fortified with $\sim 50 \mathrm{mg} / \mathrm{L}$ natamycin. These were each divided into four sub-sets (in 750 $\mathrm{mL}$ bottles) kept at $20^{\circ} \mathrm{C}$ (light and dark respectively), $30^{\circ} \mathrm{C}$ and $40^{\circ} \mathrm{C}$. Natamycin was quantified using the LC-UV method described above to study degradation kinetics. At the fortification levels used here, small injection volumes of neat wine could be employed, thereby obviating sample pretreatment. Results for one batch of red wine are shown in Fig. 4. An exponential relation was found to apply in all cases, corresponding to pseudo first-order degradation. Confirmation of the first order kinetics was obtained graphically from the linearity of plots of In concentration against time (correlation coefficients, $r$, were between 0.999 and 0.975 ). The rate constant, $k$, was calculated

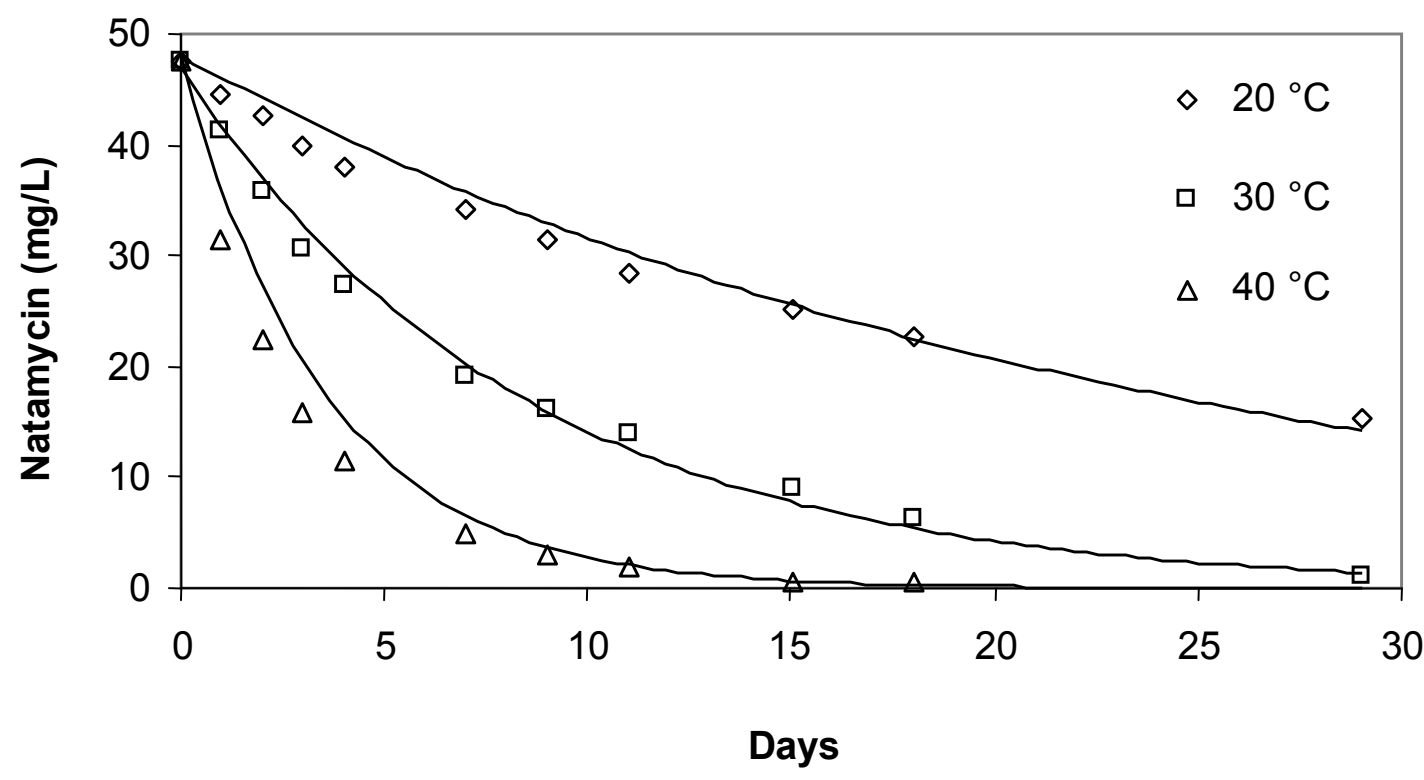

FIGURE 4

Degradation of natamycin in a red wine matrix at different temperatures. 
from the first order rate equation

$$
c_{t}=c_{0} e^{-k t}
$$

where $c_{t}$ represents the concentration of natamycin remaining at any time $t, c_{0}$ is the initial concentration and $k$ is the rate constant for the degradation reaction in days ${ }^{-1}$ (Atkins, 1998). Half-lives for each dataset were determined from the equation (Atkins, 1998)

$$
t_{1 / 2}=\frac{\ln 2}{k}
$$

The results for the degradation kinetics for natamycin in wine are summarised in Table 1 . The activation energy $\left(E_{a}\right)$ for the degradation reaction involving natamycin in wine was calculated using the Arrhenius equation (Atkins, 1998):

$$
\ln k=\ln A-\frac{E_{a}}{R T}
$$

where $A$ is the pre-exponential factor and $R$ is the gas constant $\left(=8.3143 \mathrm{~J} \cdot \mathrm{K}^{-1} \cdot \mathrm{mol}^{-1}\right)$.

Plots of $\ln k$ vs $1 / T$ provided linear relationships ( $\mathrm{r}$ between 0.994 and 1.000, Fig. 5), from which the activation energies reported in Table 2 were calculated.

These results clearly confirm that natamycin is unstable in the wine matrix under normal storage conditions. The relatively low activation energies $(\sim 80 \mathrm{~kJ} / \mathrm{mol})$ indicate that the degradation reaction proceeds readily even at normal wine storage temperatures. The half-lives for the degradation of natamycin in wine are approximately 20 days at $20^{\circ} \mathrm{C}$, six days at $30^{\circ} \mathrm{C}$ and two days at $40^{\circ} \mathrm{C}$ (Table 1). The degradation reaction proceeded faster in white wine compared to red wine, which may be ascribed to the higher acidity of the white wine
( $\mathrm{pH}$ of 3.49 compared to $\mathrm{pH} 3.55$ and 3.67 for the two red wines used here). Similar degradation rates were observed at $20^{\circ} \mathrm{C}$ in these matrices for batches stored in darkness and artificial light (results not shown). Since the samples were kept in wine bottles, radiation may have been reduced sufficiently by absorption by the glass, so that artificial light had no effect on the rate of degradation. The data reported here may then be considered representative of the stability of natamycin under normal wine storage conditions.

Several degradation products were noted in LC-UV chromatograms of natamycin in a synthetic wine matrix stored at elevated temperatures (Fig. 6). These are all more polar than natamycin, as they eluted earlier in the reversed phase separation. UV spectra of these degradation products were identical to those of intact natamycin, indicating that the degradation products retain the tetraene structure of the original molecule. This phenomenon was also noted in an earlier study (Brik, 1976). No accumulation of any specific breakdown product was noted concurrently with the degradation of natamycin in the synthetic matrix. HPLC-MS analysis of the same sample (results not shown) allowed the tentative identification of a variety of breakdown products, including mycosamine (the amino sugar degradation product of natamycin), as well as several isomers of autoxidation and hydrolysed (presumably at the epoxy group) products (Brik, 1976). Further work is required for the detailed elucidation of the relevant degradation reactions of natamycin in wine.

Factors responsible for natamycin degradation in calibration standard solutions were also investigated by evaluating stability in various matrices at $20^{\circ} \mathrm{C}$. It was found that the addition of formic acid in the range of $0(\mathrm{pH} 3.5), 1(\mathrm{pH} \mathrm{2.3)}, 5(\mathrm{pH} \mathrm{1.9})$ and $10 \%(\mathrm{pH} 1.6)$ to a series of standards prepared in $10 \%$

\begin{tabular}{|c|c|c|c|c|}
\hline Batch & $\begin{array}{l}\text { Temp. } \\
\left({ }^{\circ} \mathrm{C}\right)\end{array}$ & $\begin{array}{c}\text { Regression } \\
\text { equation }\end{array}$ & $\begin{array}{c}\text { Rate constant } \\
\text { ( } k \text {, per day) }\end{array}$ & $\begin{array}{l}\text { Half-life } \\
\left(t_{1 / 2}, \text { days }\right)\end{array}$ \\
\hline Red wine $^{\mathrm{a}}$ & 20 & $\mathrm{c}=45.4 \mathrm{e}^{-0.03868 \mathrm{t}}$ & 0.03868 & 17.9 \\
\hline White wine ${ }^{\mathrm{b}}$ & 20 & $\mathrm{c}=38.9 \mathrm{e}^{-0.05899 \mathrm{t}}$ & 0.05899 & 11.8 \\
\hline Red wine $^{c}$ & 20 & $\mathrm{c}=41.4 \mathrm{e}^{-0.03378 \mathrm{t}}$ & 0.03378 & 20.5 \\
\hline Synthetic matrix ${ }^{\mathrm{d}}$ & 20 & $\mathrm{c}=52.8 \mathrm{e}^{-0.01443 \mathrm{t}}$ & 0.01443 & 48.0 \\
\hline Red wine $^{\mathrm{a}}$ & 30 & $\mathrm{c}=45.1 \mathrm{e}^{-0.1202 \mathrm{t}}$ & 0.1202 & 5.8 \\
\hline White wine ${ }^{\mathrm{b}}$ & 30 & $\mathrm{c}=35.9 \mathrm{e}^{-0.1592 \mathrm{t}}$ & 0.1592 & 4.4 \\
\hline Red wine $^{\mathrm{c}}$ & 30 & $\mathrm{c}=45.5 \mathrm{e}^{-0.1216 \mathrm{t}}$ & 0.1216 & 5.7 \\
\hline Synthetic matrix ${ }^{d}$ & 30 & $\mathrm{c}=52.4 \mathrm{e}^{-0.03605 \mathrm{t}}$ & 0.03605 & 19.2 \\
\hline Red wine $^{\mathrm{a}}$ & 40 & $\mathrm{c}=40.0 \mathrm{e}^{-0.2861 \mathrm{t}}$ & 0.2861 & 2.4 \\
\hline White wine $^{\mathrm{b}}$ & 40 & $\mathrm{c}=33.8 \mathrm{e}^{-0.4368 \mathrm{t}}$ & 0.4368 & 1.6 \\
\hline Red wine $^{\mathrm{c}}$ & 40 & $\mathrm{c}=37.1 \mathrm{e}^{-0.2735 \mathrm{t}}$ & 0.2735 & 2.5 \\
\hline Synthetic matrix ${ }^{\mathrm{d}}$ & 40 & $\mathrm{c}=53.7 \mathrm{e}^{-0.1151 \mathrm{t}}$ & 0.1151 & 6.0 \\
\hline
\end{tabular}

TABLE 1

Kinetic parameters for the degradation of natamycin in wine $(n=4)$.

${ }^{\mathrm{a}} \mathrm{pH}=3.55 \quad{ }^{\mathrm{b}} \mathrm{pH}=3.49 \quad{ }^{\mathrm{c}} \mathrm{pH}=3.67 \quad{ }^{\mathrm{d}} \mathrm{pH}=3.50$ 
methanol caused a drastic acceleration in the degradation of the compound. However, the acidification (minimum of $0.5 \%$ formic acid with $40 \%$ methanol in water) of standards is indispensable to ensure solubility in solvents equivalent to the starting conditions of reversed phase separation. Natamycin is soluble at $\sim 50 \mu \mathrm{g} / \mathrm{mL}$ in water (Pedersen, 1992). Moreover, it was found that natamycin stability increased with methanol content in a series of standards prepared in 5, 10, 20 and $40 \%$ methanol (constant acidity). These findings are consistent with a predominantly hydrolytic degradation mechanism, as degradation is generally faster at lower $\mathrm{pH}$ and higher aqueous content respectively. For these reasons, we prepared all standard solutions in $40 \%$ methanol and $0.5 \%$ formic acid in water. Standard solutions were stored at $4^{\circ} \mathrm{C}$, while new standards were prepared monthly.

\section{CONCLUSIONS}

Natamycin is not a natural constituent of wine and should therefore be absent from the product where it is prohibited. The presence of natamycin in wine may be due to direct addition for preservation purposes (for example in countries where this practice is allowed), or as a result of secondary contamination. Sources of secondary contamination include the addition of sweet must to the product, as well as contamination during the processing, bottling and ageing of the product (for example, natamycin was detected in some imported European compound corks). Local wine producers should be made aware of this risk and take all possible measures to avoid possible sources of secondary contamination in wines intended for export. Although the resulting levels of natamycin would be below the effective concentration of the substance, it would nevertheless

TABLE 2

Parameters for the determination of the activation energy $\left(E_{a}\right)$ for the degradation of natamycin in wine.

\begin{tabular}{ccccc}
\hline Batch & Temp. $\left({ }^{\circ} \mathbf{C}\right)$ & $\boldsymbol{l n} \boldsymbol{k}$ & $\mathbf{1 / T}(\mathbf{p e r} \mathbf{K})$ & Activation energy, $E_{a}(\mathbf{k J} / \mathbf{m o l})$ \\
\hline Red wine & 20 & -3.252 & 0.00341 & 76 \\
& 30 & -2.119 & 0.00330 & 76 \\
White wine & 40 & -1.251 & 0.00319 & \\
& 20 & -2.830 & 0.00341 & 79 \\
Red wine & 30 & -1.838 & 0.00330 & \\
& 40 & -0.8283 & 0.00319 & \\
& 20 & -3.388 & 0.00341 & 78 \\
\hline
\end{tabular}

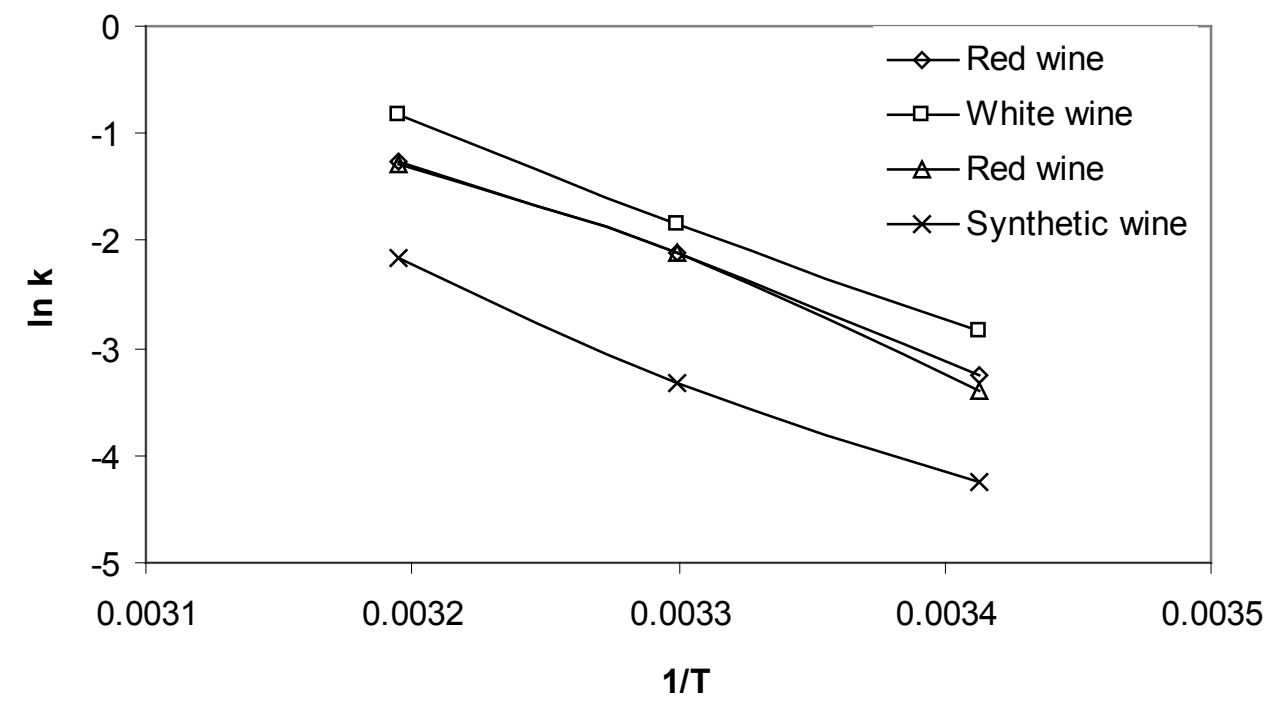

FIGURE 5

Arrhenius plots for the degradation of natamycin in wine matrices. 


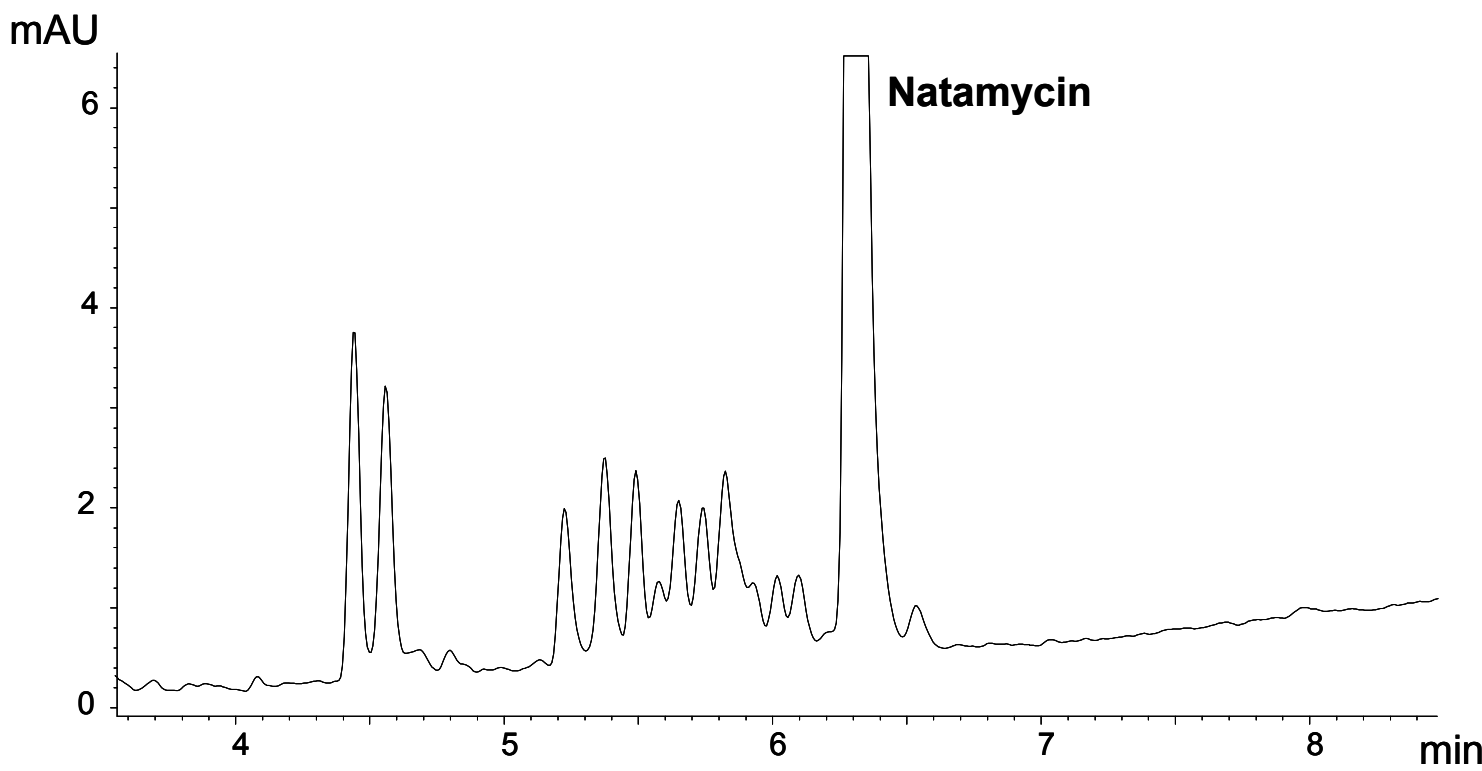

FIGURE 6

LC-UV chromatogram of the analysis of natamycin in a synthetic wine matrix stored for 18 days at $30^{\circ} \mathrm{C}$, illustrating the degradation products.

result in the rejection of products intended for the European market.

In terms of the analytical quantitation of natamycin in wine, the latter condition clearly places a high demand on method sensitivity. The WHO regards exposure to antimicrobials at sub-effective levels as a contributing factor in the spread of antimicrobial resistance (World Health Organization, 2002), while German authorities implement acceptance levels of $<$ $0.005 \mathrm{mg} / \mathrm{L}$ natamycin in wine. Suitable analytical methods are therefore required to demonstrate compliance and facilitate the international wine trade. Another relevant requirement is for methods to be sufficiently fast and robust for the highthroughput, routine analysis of large numbers of samples.

In this study, methods based on HPLC analysis in combination with SPE sample cleanup were developed for the determination of natamycin in wine. LC-UV is accurate, robust and inexpensive, although not sufficiently sensitive to demonstrate compliance with EU standards. The LC-MS/ MS procedure developed in this study is very sensitive and also provides qualitative information for positive compound identification. The use of dedicated ultra-high pressure columns and instrumentation provides very rapid analyses. In combination with an optimised SPE method, the overall procedure is fast and sufficiently specific to be rendered free from interference by the wine matrix. The developed methodology therefore produces performance specifications well within the requirements and is ideally suited for routine application to demonstrate compliance of wines with EU directives concerning natamycin.

Furthermore, degradation studies have revealed that natamycin decomposes readily under normal wine storage conditions. Half-lives are of the order of 20 days at $20^{\circ} \mathrm{C}$, and the activation energy for the decomposition is $\sim 80 \mathrm{~kJ} / \mathrm{mol}$. The fact that the substance is labile in the wine matrix complicates accurate quantitation, since time as well as storage conditions (mainly temperature) affect its concentration. Aqueous calibration standards are also unstable and precautions such as cold storage and the regular preparation of fresh standards are required for accurate quantitation.

\section{LITERATURE CITED}

Abad-Garcia, B., Berrueta, L.A., Garmon-Lobato, S., Gallo, B. \& Vicente, F., 2009. A general analytical strategy for the characterization of phenolic compounds in fruit juices by high-performance liquid chromatography with diode array detection coupled to electrospray ionization and triple quadrupole mass spectrometry. J. Chromatogr. A 1216, 5398-5415.

Atkins, P.W., 1998 (6 $6^{\text {th }}$ ed). Physical chemistry. Oxford University Press, Oxford.

Brik, H., 1976. New high-molecular decomposition products of natamycin (pimaricin) with intact lactone-ring. J. Antibiotics 29, 632-636.

Budavari, S., 1989 (11 ${ }^{\text {th }}$ ed). The Merck Index: An encyclopaedia of chemicals, drugs and biologicals. Merck \& Co. Inc., Rahway, NJ.

Capitan-Vallvey, L.F., Checa-Moreno, R. \& Navas, N., 2000. Rapid ultraviolet spectrophotometric and liquid chromatographic methods for the determination of natamycin in lactoserum matrix. J. AOAC Int. 83, 802-808.

De Ruig, W., Van Oostrom, J.J. \& Leenheer, K., 1987. Spectrometric and liquid chromatographic determination of natamycin in cheese and cheese rind. J. Assoc. Off. Anal. Chem. 70, 944-948.

De Villiers, A., Lestremau, F., Szucs, R., Gélébart, S., David, F. \& Sandra, P., 2006. Evaluation of ultra performance liquid chromatography. Part I: Possibilities and limitations. J. Chromatogr. A, 1127, 60-69.

Food Standards Australia New Zealand (FSANZ), October 2004. Application A542, Natamycin - extension of use as a food additive.

Koontz, J.L., Marcy, J.E., Barbeau, W.E. \& Duncan, S.E., 2003. Stability of natamycin and its cyclodextrin inclusion complexes in aqueous solution. J. Agric. Food Chem. 51, 7111-7114.

Pedersen, J.C., 1992. Applied \& Environmental Microbiology 58, 1064-1066. 
Republic of South Africa, 1989. Liquor Products Act, Act 60. Government Gazette of the Republic of South Africa, Pretoria.

Thurman, E.M. \& Mills, M.S., 1998. Solid-phase extraction. Principles and practice. Chemical analysis: A series of monographs on analytical chemistry and its applications, vol. 147. John Wiley \& Sons, New York.
World Health Organization, January 2002. Fact sheet No. 194. WHO, Geneva, Switzerland. 